\title{
Editorial
}

\section{Nanomaterials for Sensing Applications}

\author{
Wen Zeng, ${ }^{1}$ Hua Wang, ${ }^{2}$ and Zhenyu $\mathrm{Li}^{3}$ \\ ${ }^{1}$ College of Materials Science and Engineering, Chongqing University, Chongqing 400040, China \\ ${ }^{2}$ School of Chemistry and Environment, Beihang University, Beijing 100083, China \\ ${ }^{3}$ Institute for Frontier Materials, Deakin University, Geelong, VIC 3216, Australia
}

Correspondence should be addressed to Wen Zeng; wenzeng@cqu.edu.cn

Received 12 June 2016; Accepted 13 June 2016

Copyright (C) 2016 Wen Zeng et al. This is an open access article distributed under the Creative Commons Attribution License, which permits unrestricted use, distribution, and reproduction in any medium, provided the original work is properly cited.

Sensor played a huge role in the field of preventing the fire explosion accident, atmospheric environmental testing, and the industrial production of poisonous and harmful gas detection. However, at present the development of sensitive material is still obvious deficiencies in selectivity, stability, and other aspects; therefore, the research and development of new high performance sensors will have very important realistic meaning and practical value.

The performance of sensing materials depends critically on their microstructures, which requires the development of materials processing techniques to obtain the desired microstructures and morphologies. It is of great importance in future technological applications to understand how the materials behave at nanoscale during working and how controllably are manufactured. No matter physical or chemical preparation, forward-looking theoretical guidance and characterization proof are necessary for explaining the formation mechanism so as to design devices with expected sensing properties. In this issue, different approaches were used to create nanostructures in several nanomaterials to deal with the sensing application.

In the paper "Hydrothermal Synthesis and Responsive Characteristics of Hierarchical Zinc Oxide Nanoflowers to Sulfur Dioxide" Q. Zhou et al. prepare hierarchical ZnO nanoflowers through a simple hydrothermal process and report systematically their responsive characteristics to $\mathrm{SO}_{2}$ and demonstrate that the sensor fabricated with hierarchical $\mathrm{ZnO}$ nanoflowers is a promising candidate for detecting $\mathrm{SO}_{2}$. In the paper "Structural and Electrical Studies on $\mathrm{ZnO}$ Based Thin Films by Laser Irradiation" S. Zhao et al. studied the structural and electrical properties of the $\mathrm{ZnO}$-based thin film varistors for low-voltage application. They found that the pressure-sensitive electrical properties of $\mathrm{ZnO}$-based ceramic film of laser irradiation state show excellent property. In the paper "Amorphous Silicon-Germanium Films with Embedded Nanocrystals for Thermal Detectors with Very High Sensitivity" C. Calleja et al. optimized the deposition conditions of amorphous silicon-germanium films with embedded nanocrystals in a plasma enhanced chemical vapor deposition (PECVD) reactor, working at a standard frequency of $13.56 \mathrm{MHz}$. The results show optimized deposition conditions of amorphous silicon-germanium films with embedded nanocrystals in a plasma enhanced chemical vapor deposition (PECVD) reactor, working at a standard frequency of $13.56 \mathrm{MHz}$.

In the paper "Nanosensing Backed by the Uncertainty Principle" I. Filikhin et al. studied spectral distributions of localized/delocalized states of a single electron in a double quantum well (DQW) with relation to slight asymmetry perturbations and demonstrated that the DQW is a quantum system suitable for sensing tiny substances adsorbed on one of the quantum dots constituting the DQW or by a defect in one of the dots.

The paper "Research on Acetylene Sensing Properties and Mechanism of $\mathrm{SnO}_{2}$ Based Chemical Gas Sensor Decorated with $\mathrm{Sm}_{2} \mathrm{O}_{3}$ " by Q. Zhou et al. reports the successful preparation and characterization of samarium oxide $\mathrm{Sm}_{2} \mathrm{O}_{3}$ decorated tin oxide $\mathrm{SnO}_{2}$ based sensors with hierarchical rod structure for $\mathrm{C}_{2} \mathrm{H}_{2}$ gas detection. The obtained materials show an excellent gas sensing performance.

In the paper "Optical and Sensing Properties of $\mathrm{Cu}$ Doped ZnO Nanocrystalline Thin Films" R. K. Shukla et al. synthesized nanocrystalline $\mathrm{Cu}$ doped $\mathrm{ZnO}$ films deposited on glass substrates by spray pyrolysis. They found that 
the crystallite size decreases with increase in molarity of precursor solution whereas with doping the crystallite size increases in general. Doping increases the transmittance of the films whereas optical band gap first decreases and then increases as the dopant concentration is gradually increased for both molarities in reference.

By compiling these papers, we hope that this issue will provide some valuable insight to our readers and researchers with respect to field of nanomaterials for sensing applications.

Wen Zeng

Hua Wang

Zhenyu Li 

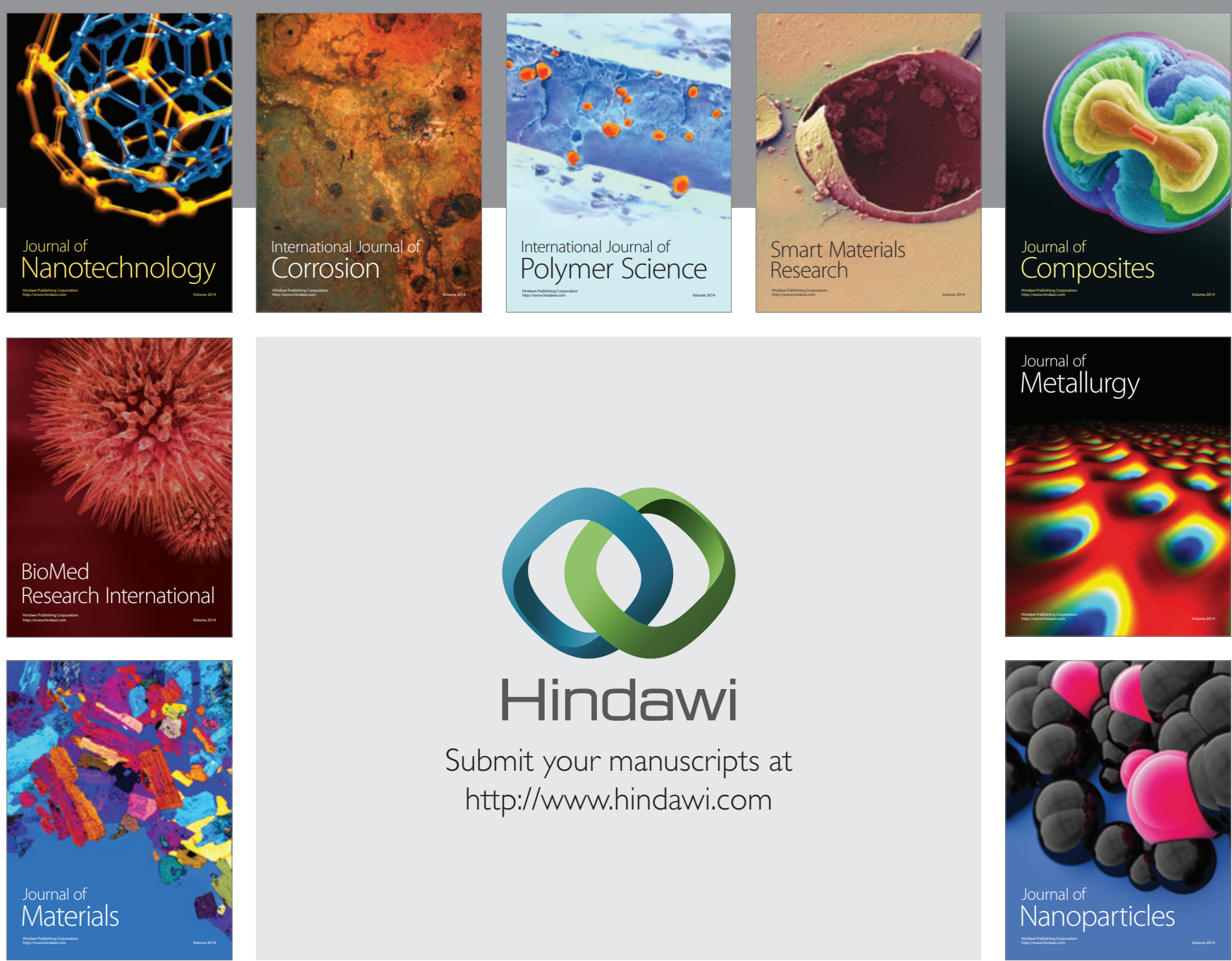

\section{Hindawi}

Submit your manuscripts at

http://www.hindawi.com

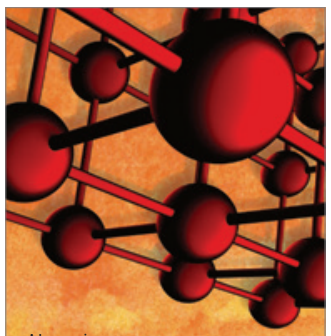

Materials Science and Engineering
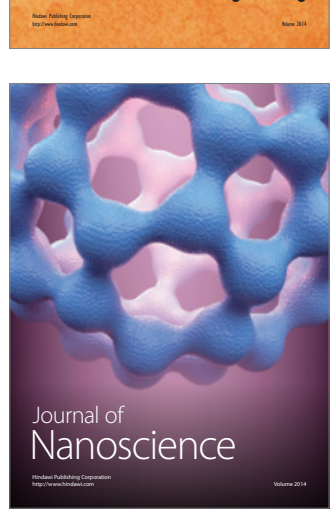

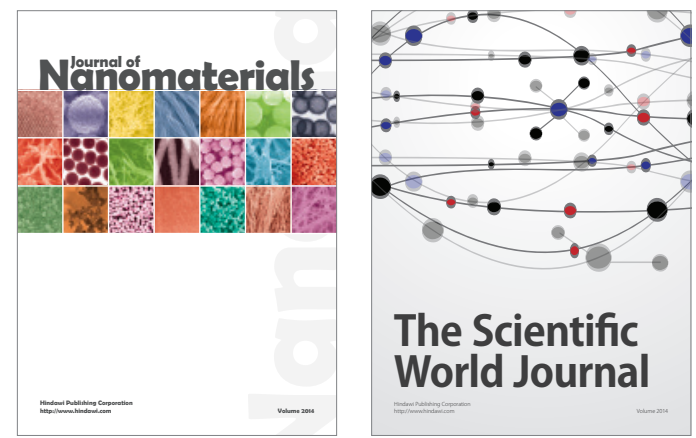

The Scientific World Journal
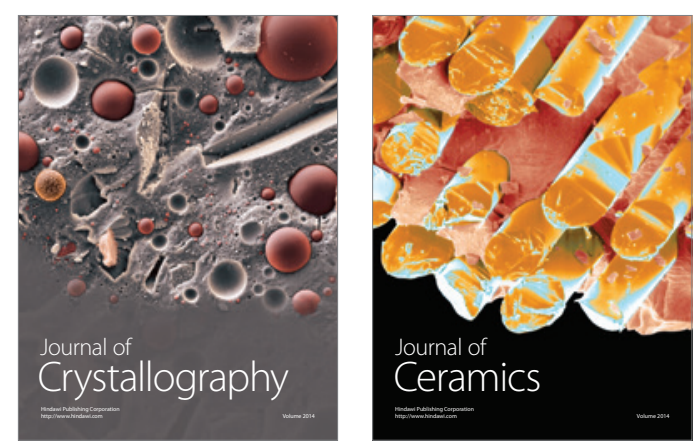
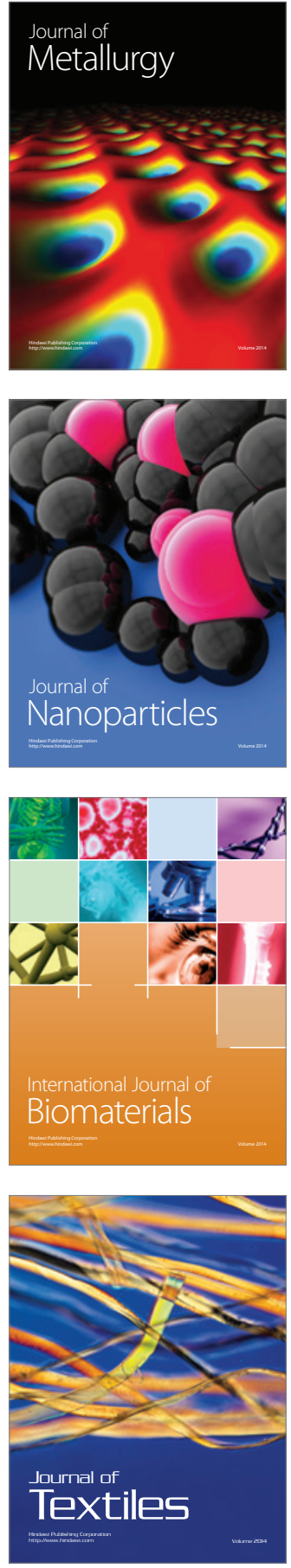